\title{
Improving compliance to controlled drug prescription guidelines through the introduction of an EMIS template
}

\author{
Authors: Pratap Harbham, ${ }^{\mathrm{A}}$ Ahmed Hussain, ${ }^{\mathrm{A}}$ Darshana Nair ${ }^{\mathrm{A}}$ and Abdul Kutty ${ }^{\mathrm{A}}$
}

\section{Introduction}

Due to their significant potential for misuse and harm, controlled drug prescriptions are required to fulfil specific legal requirements. In particular, it is essential to clearly document reasons for issuing controlled drug prescriptions for more than 28 days and discussions around the adverse effects of controlled drugs, and these requirements have medico-legal implications for practitioners. We aimed to audit baseline documentation adequacy around controlled drug prescriptions and improve any inadequacies found through a two-stepped intervention.

\section{Materials and methods}

In September 2018 baseline data from all patients issued a controlled drug prescription in the previous 2 months were collected, capturing demographic data and documentation of discussions of the adverse effects of controlled drugs. Patients prescribed a controlled drug for longer than 28 days were further surveyed for clearly documented indications for this. Following this a two-stepped intervention, comprising an educational session and the introduction of a controlled-drug prescribing EMIS template, were implemented. A re-audit was performed in February 2019.

\section{Results and discussion}

Baseline data showed that of 20 patients prescribed controlled drugs for more than 28 days, clear documentation was present for 0 ; documentation of discussion of adverse effects present in 4 of 163 patients. Post-intervention, 3 of 12 patients had clearly documented reasons for prescriptions longer than 28 days (significant: chi-squared $=5.517 ; p=0.0188$ ). Adverse effects documentation post-intervention was 24 of 154 (significant: chi-squared $=16.345 ; p=0.0001$ ).

\section{Conclusion}

The study highlights patient safety and medico-legal implications for practices with inadequate documentation of controlled drug prescriptions. Our intervention showed significant improvement in documentation adequacy and represents a quick and efficient method for improving controlled drug prescription practices that is reproducible in different settings.

\section{Conflicts of interest}

None declared.

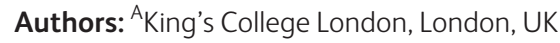

